\title{
Linkage of the Angiotensinogen Gene Locus to Human Essential Hypertension in African Caribbeans
}

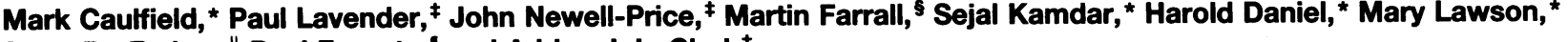 \\ Perry De Freitas," Paul Fogarty, ' and Adrian J. L. Clark ${ }^{\ddagger}$ \\ Departments of *Clinical Pharmacology and ${ }^{\ddagger}$ Chemical Endocrinology, St. Bartholomew’s Hospital, London EC1A 7BE, United \\ Kingdom: ${ }^{8}$ The Wellcome Trust Centre for Human Genetics, Oxford OX3 7BN, United Kingdom; "The Ministry of Health of St. Vincent \\ and the Grenadines, West Indies; and 'St. Vincent's Hospital, Melbourne, Victoria 3065, Australia
}

\begin{abstract}
The renin-angiotensin system regulates blood pressure and sodium balance. The angiotensinogen gene which encodes the key substrate within this system has been linked to essential hypertension in White Europeans. It has been suggested that people of West African ancestry may have a different genetic basis for hypertension. In this study we have tested whether there is linkage of the angiotensinogen gene to essential hypertension in African Caribbeans from St. Vincent and the Grenadines.
\end{abstract}

DNA from 63 affected sibling pairs with hypertension was tested for linkage by analyzing whether there was excess allele sharing among siblings genotyped using an angiotensinogen dinucleotide repeat sequence. There was significant support for linkage $(T=3.07, P=0.001)$ and association of this locus to hypertension $\left(\chi^{2}=50.2,12\right.$ degrees of freedom, $P \ll 0.001)$. A DNA polymorphism which alters methionine to threonine at position $235(M 235 T)$ within the angiotensinogen peptide has been associated previously with hypertension. However, we found no association of this variant with hypertension in this study.

These findings provide support for linkage and association of the angiotensinogen locus to hypertension in African Caribbeans and suggest some similarities in the genetic basis of essential hypertension in populations of different ethnicity. (J. Clin. Invest. 1995. 96:687-692.) Key words: angiotensinogen • hypertension • genetics - African Caribbeans

\section{Introduction}

Human essential hypertension is a multifactorial disorder arising from the influence of several susceptibility genes and environmental stimuli (1). There is evidence suggesting that genes may contribute to $30 \%$ of the variation of blood pressure. However, the number of genes involved or the model of interaction with other genes or environmental risk factors is unknown (1).

P. Lavender and M. Caulfield contributed equally to this work.

Address correspondence to Dr. Mark Caulfield, Department of Clinical Pharmacology, St. Bartholomew's Hospital, London EC1A 7BE, United Kingdom. Phone: 171-601-7423; FAX: 171-601-8134. Paul Lavender's current address is The Wellcome CRC Institute, Tennis Court Rd., Cambridge, United Kingdom.

Received for publication 14 December 1994 and accepted in revised form 12 April 1995.

J. Clin. Invest.

(C) The American Society for Clinical Investigation, Inc.

0021-9738/95/08/0687/06 \$2.00

Volume 96, August 1995, 687-692
Recently, the angiotensinogen gene which encodes a key component within the renin-angiotensin system has been linked to essential hypertension in two separate studies of populations of White European ancestry $(2,3)$. A variant within this gene, encoding threonine rather than methionine at position 235 (M235T), has been associated with hypertension in one of these studies (3).

The greater prevalence of hypertension in migrant populations of African ancestry and observations of higher rates of end organ complications, in terms of stroke and renal disease, have generated speculation that there may be a different genetic basis for hypertension among these groups (4-6). Physiologi$\mathrm{cal}$ and pharmacological evidence suggesting that African $\mathrm{Ca}-$ ribbean hypertensives have low-renin, sodium-sensitive blood pressure and respond less well to treatment with angiouensin converting enzyme inhibitors have lent support to this argument $(7,8)$.

In the investigation of the genetic basis of polygenic disorders such as essential hypertension it is critical to be able to test for linkage of candidate genes in several populations with different ethnicity. In view of the heterogeneity of British populations of African ancestry, we tested whether there is linkage of the angiotensinogen gene to essential hypertension in affected sibling pairs of West African origin from St. Vincent and the Grenadines.

\section{Methods}

Subjects. After ethical approval and permission from the Ministry of Health of St. Vincent and the Grenadines, fully equipped, trained field teams were sent from the United Kingdom. All subjects were of African ancestry as defined by grandparental and parental ethnicity and were identified via the primary care clinic network on the island of St. Vincent. The teams measured blood pressure in accordance with the 1986 British Hypertension Society guidelines (9) and identified 63 families with at least 1 affected sibling pair. These affected relatives had sitting diastolic pressures $>95 \mathrm{mmHg}$ or were receiving treatment for essential hypertension and had documentary evidence of diastolic pressures $>95$ $\mathrm{mmHg}$. Families with relatives with secondary hypertension and diabetes were excluded.

150 unrelated individuals with essential hypertension and a recorded family history of parental or sibling hypertension were defined by blood pressure $>95 \mathrm{mmHg}$, or by receipt of antihypertensive therapy with documentary evidence of blood pressures $>95 \mathrm{mmHg}$ diastolic. All individuals with clinical evidence of secondary hypertension or diabetes were excluded. 93 unrelated normotensive controls with a negative family history of parents or siblings with hypertension and diastolic blood pressure $<85 \mathrm{mmHg}$ were also identified from the same primary care clinics.

A random control population of 49 individuals to provide population-based allele frequencies was recruited.

The field teams recorded family pedigrees, age, body mass index, alcohol consumption in units per week, smoking habit, blood pressure, 
blood glucose (using a glucometer from Boehringer-Mannheim, Mannheim, Germany), and current treatment. All subjects were recruited through semirural primary care clinics and hypertensive sibling pairs, hypertensive unrelated cases, normotensive, and random controls were drawn from the same population. Genomic DNA was extracted from whole blood taken into potassium EDTA tubes using phenol-chloroform purification after transfer to the United Kingdom.

Angiotensinogen GT repeat analysis. A highly polymorphic dinucleotide $G T$ repeat located in the $3^{\prime}$ flanking region of the angiotensinogen gene was used to test for linkage (10) and was analyzed by methods we have described previously (2). After polymerase chain reaction amplification of genomic DNA, radioactive products were analyzed by electrophoresis through $5 \%$ denaturing polyacrylamide gels containing $8 \mathrm{M}$ urea, $89 \mathrm{mM}$ Tris base, $89 \mathrm{mM}$ boric acid, and $2 \mathrm{mM}$ EDTA (Sigma Ltd., Dorset, United Kingdom). Gels were run for $4 \mathrm{~h}$ at a constant voltage of $1,350 \mathrm{~V}$, dried, and exposed over $12 \mathrm{~h}$ to Kodak XAR-2 film for autoradiography (11) (Sigma Ltd.). All autoradiographs were genotyped by two independent observers. Hypertensives, sibling pairs, normotensives, and random population controls were run together to further cross-check allele sizes.

M235T analysis. The variant $M 235 T$ was analyzed after polymerase chain reaction amplification of genomic DNA and restriction digestion with Tth111I (Promega, Southampton, United Kingdom) using the method described by Russ (12). In the antisense primer two nucleotides are mismatched with the genomic DNA which, depending on the sequence, creates a Th111I restriction site during amplification. If the codon is ACG ( $T 235)$, restriction digestion at $65^{\circ} \mathrm{C}$ with Tth111I yields a product of $141 \mathrm{bp}$ relative to the undigested 165-bp fragment (M235) (12). Genotyping of $M 235 T$ was undertaken after size fractionation on $8 \%$ nondenaturing polyacrylamide gels and ethidium bromide staining. This particular method was chosen because of reports of high frequencies of $T 235$ in populations of African ancestry (13).

Statistical methods. The affected pedigree member (APM) ${ }^{1}$ method is an established method of linkage analysis which is particularly applicable for genetic investigation of complex disorders like essential hypertension (2, 14-17). The advantages of this method in hypertension are that no assumptions are made regarding mode of inheritance, there is no requirement for parental genotype, and the method can take account of variable penetrance by causative genes $(14,15)$.

In this study we used the APM method to compute a $T$ statistic which tests whether affected relatives share alleles of the angiotensinogen $G T$ repeat more often than would be expected by chance in all 63 families (14, 15). The $T$ statistics derived are weighted to allow the excess sharing of rare alleles to outweigh the importance of common alleles. The recommended intermediate weighting function $1 /$ sqrt $(p)$, where $\mathrm{p}$ denotes the control allele frequency, was used in this study $(14,15)$. For the APM method we used the $G T$ repeat distribution in the 49 random controls to define population allele frequencies. The 63 families were further tested for an influence of gender upon the support for linkage by dividing the families into male-male and female-female pairs and testing the subgroups for linkage using the APM method.

This $T$ statistic is a measure of excess allele sharing and therefore can only be interpreted as a single-tailed test. Additionally, this statistic is sensitive to numbers analyzed and accordingly should not be used as a direct measure for strength of linkage. As an indicator of strength of linkage in our sibships we include the percentage of excess alleles shared. This is computed by using the equation indicated below to compare the sum of the observed identity by state scores for allele sharing with the sum of the expected scores and expressing this as the percentage of excess alleles shared.

$\%$ excess alleles shared $+1=\frac{\sum_{1}^{N} \text { observed IBS score }}{\sum_{1}^{N} E(\text { IBS score })}$

where $N$ is the number of families and $I B S$ is the identity by state.

1. Abbreviation used in this paper: APM, affected pedigree member.
Table I. Demographic Characteristics of the 63 Families, Consisting of 130 Individuals (100 Affected Females and 30 Affected Males)

\begin{tabular}{lcc}
\hline \multicolumn{1}{c}{ Variable } & Median & Interquartile range \\
\hline Age (yr) & 65 & $58-71$ \\
Body mass index* & 27.1 & $23.7-31.3$ \\
Alcohol consumption (units/wk) & 0 & $0-3$ \\
Systolic blood pressure $(\mathrm{mmHg})$ & 177 & $160-190$ \\
Diastolic blood pressure $(\mathrm{mmHg})$ & 100 & $100-110$ \\
\hline
\end{tabular}

* Measured in kilograms divided by the height in meters squared. ${ }^{\ddagger} 1$ unit of alcohol equals 15 grams.

The results obtained hint at the strength of support for linkage in a group of families, however, this method is influenced by family structures and it may be misleading to compare the percentage of excess alleles shared between different data sets.

To test for complimentary support for the linkage data we tested the angiotensinogen $G T$ repeat for association in 150 unrelated hypertensive cases and 93 unrelated normotensive controls using the $\chi^{2}$ test. The 63 hypertensive index cases from each sibship were also compared with the 93 normotensive controls in the same manner. Entire families cannot be used for this analysis as they will share alleles by virtue of their relationship.

The variant $M 235 T$ was genotyped in the 63 index cases from the hypertensive families and 150 unrelated hypertensives which were compared with 93 normotensive controls and tested for association with hypertension using the $\chi^{2}$ test.

\section{Results}

63 families including 130 affected relatives were identified from 12 primary care clinics on the island of St. Vincent and were genotyped blind using the angiotensinogen $G T$ repeat (10). The demographic characteristics of these families are summarized in Table I. Control allele frequencies were determined from a random population of 49 individuals (Fig. 1).

The distribution of the $G T$ repeat alleles among Vincentian controls was very different to the frequencies previously reported in 64 White European controls (2) as indicated in Fig. 1. In particular we observed 13 alleles instead of the 11 alleles previously described in White Europeans with a polymorphic information content of 0.85 . The extra alleles are numbered -1 and -2 in order to make comparison of the distribution in other studies possible (see Figs. 1 and 3) $(2,3,10)$.

Linkage was detected in the 63 sibships $(T=3.07, P$ $=0.001$ ) and 14 families shared $29 \%$ alleles in excess. The alleles shared in excess among these families include alleles 9 and 10 which are different from those we have reported previously for White European families (2). The variance between the observed and expected distribution of the family by family $T$ statistics can be seen for all 63 families (Fig. 2). This demonstrates a positive skew to the right of the observed $T$ statistic supporting linkage of angiotensinogen to hypertension in some of the Vincentian families.

When the 63 families were partitioned according to gender there were 46 female-female pairs and 6 male-male pairs reflecting the predominance of female sex in the 63 families. There was support for linkage observed in the 46 female-female pairs $(T=3.82, P \leq 0.001)$ who exhibited $40 \%$ excess allele 


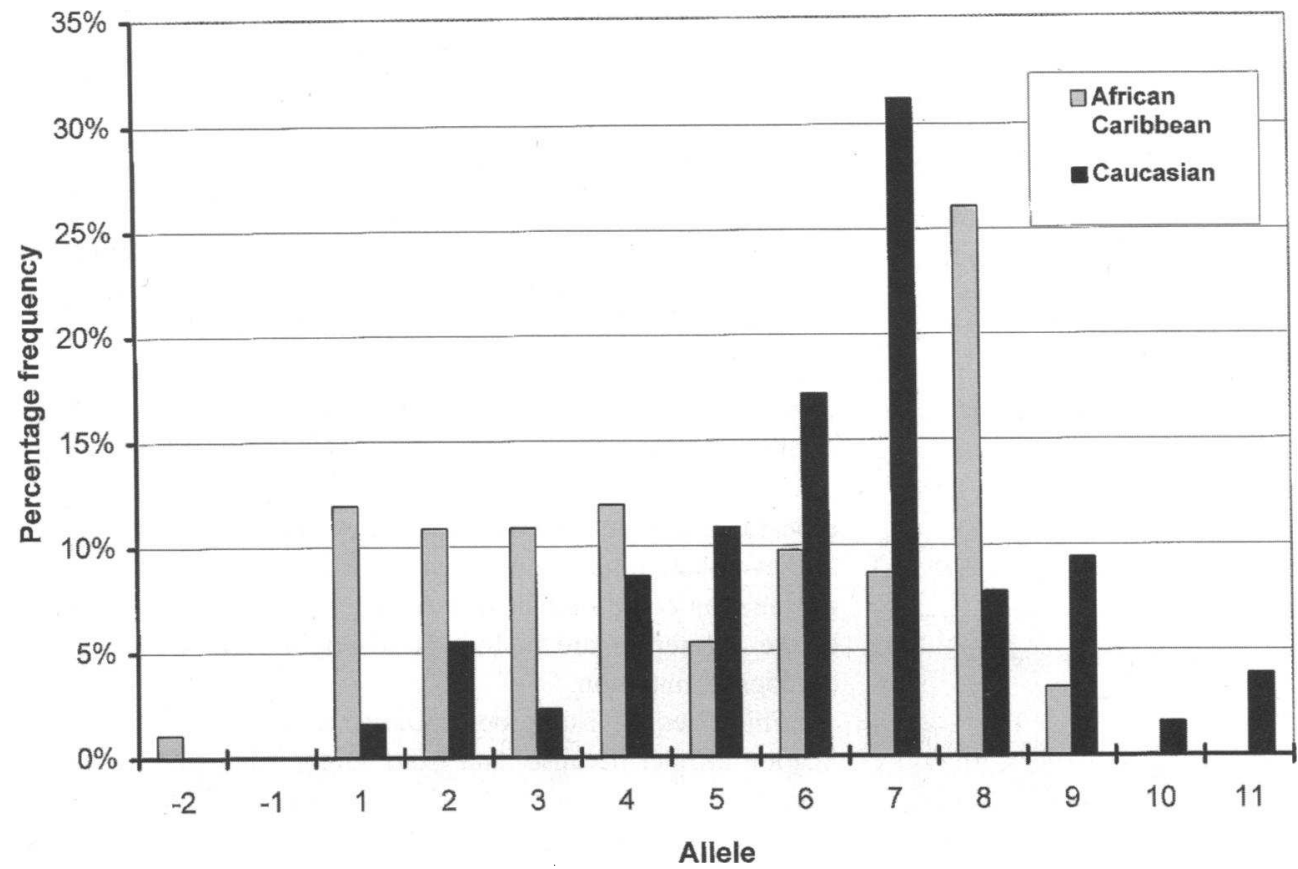

Figure 1. The distribution of the angiotensinogen $G T$ repeat alleles in 49 African Caribbeans from St. Vincent compared with 64 White European controls from the United Kingdom (2). There were 13 alleles observed in the African Caribbeans and 11 in the White Europeans. sharing. However, there was no support for linkage among six male-male pairs $(T=0.78, P \geq 0.05)$ which may reflect the size of the data set.

Substratification according to those with body mass index below the median or by gender did not contribute to the analysis because of the small number of families available. Unfortunately, the distribution of blood pressures within families did not permit stratification by severity of systolic or diastolic blood pressure.

To examine further the role of the angiotensinogen locus in hypertension an additional 150 unrelated hypertensive individu- als and 93 normotensive controls were genotyped to test for association of alleles of the GT repeat with hypertension (demographic data in Table II). Although there is an age difference between cases and controls, both groups were recruited from decades in which hypertension is most likely to be manifest and from the same primary care clinics. Fig. 3 shows a difference in the distribution of the genotypes in the two groups which was confirmed by association testing $\left(\chi^{2}=50.2,12\right.$ degrees of freedom [d.f.], $P=0.000001$ ). The relative overrepresentation of alleles 9 and 10 and underrepresentation of alleles 5 and 8 are clearly demonstrated. The allelic distribution from the 63

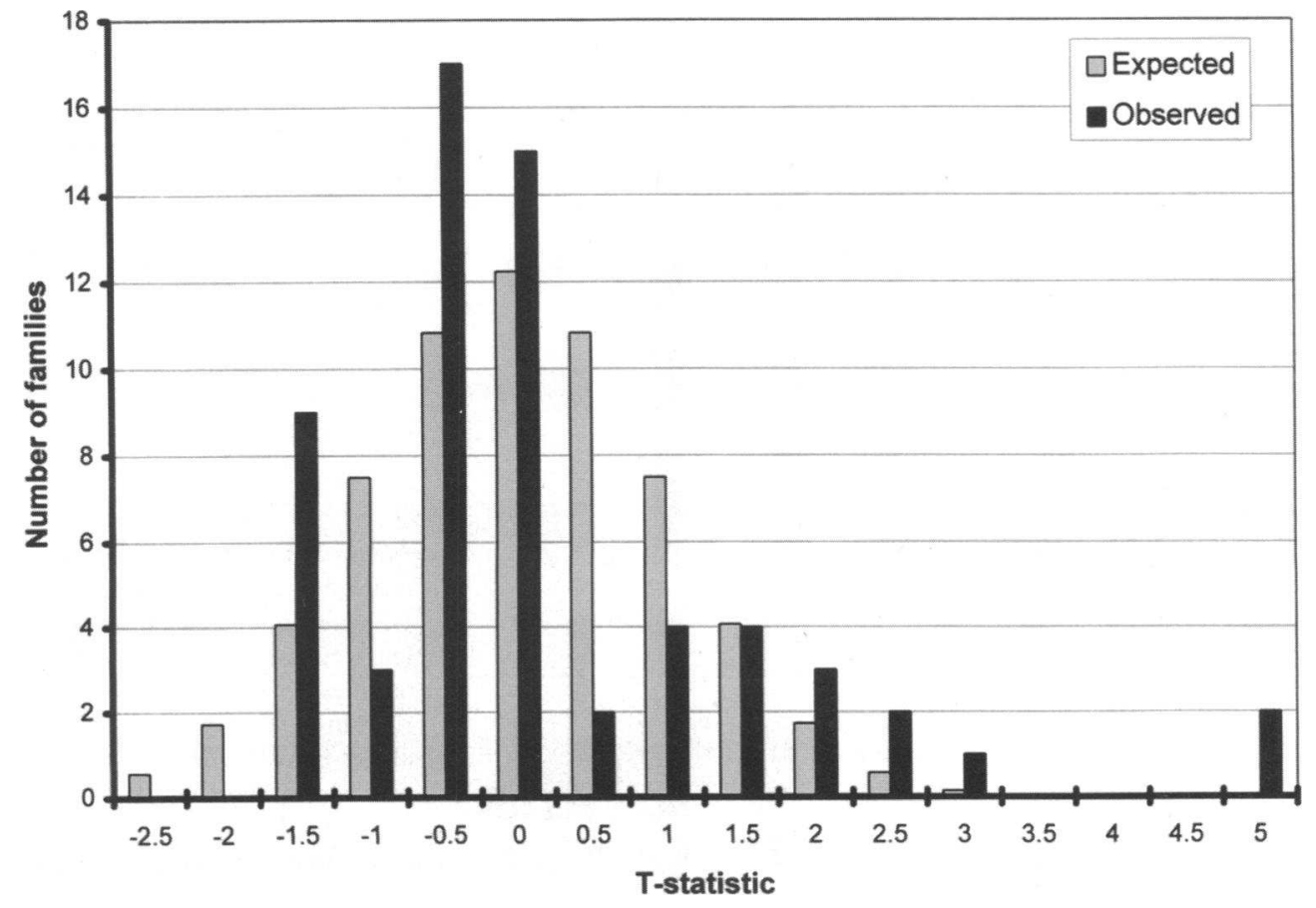

Figure 2. The distribution of the observed and expected family by family $T$ statistic for all 63 African Caribbean families. This shows a positive skew of the observed $T$ statistic indicating support for linkage in some Vincentian families. 
Table II. Demographic Data for Association Study of Hypertensives Compared with Normotensives Expressed as the Median (Interquartile Range)

\begin{tabular}{lcccc}
\hline \multicolumn{1}{c}{ Variable } & \multicolumn{1}{c}{$\begin{array}{c}\text { Hypertensives } \\
(n=150)\end{array}$} & $\begin{array}{c}\text { Normotensives } \\
(n=93)\end{array}$ \\
\hline $\begin{array}{l}\text { Age }(\mathrm{yr}) \\
\text { Body mass index } \\
\left(\mathrm{kg} / \mathrm{m}^{2}\right)^{*}\end{array}$ & 64 & $(56-72)$ & 54 & $(40-62)$ \\
$\begin{array}{c}\text { Alcohol consumption } \\
(\text { units/wk) }\end{array}$ & $26.42(23.1-31.3)$ & 24.9 & $(21.9-27.4)$ \\
$\begin{array}{l}\text { Systolic blood pressure } \\
(\mathrm{mmHg})\end{array}$ & 0 & $(0-3)$ & 0 & $(0-2)$ \\
$\begin{array}{c}\text { Diastolic blood pressure } \\
(\mathrm{mmHg})\end{array}$ & 175 & $(160-190)$ & 130 & $(120-130)$ \\
\hline
\end{tabular}

* Measured in kilograms divided by the height in meters squared. ${ }^{\ddagger} 1$ unit of alcohol equals 15 grams.

index cases from our families indicates similar departure from the normotensive frequencies $\left(\chi^{2}=34.75,11\right.$ d.f., $P=$ $0.00004)$.

The distribution of genotypes for M235T was in HardyWeinberg equilibrium and revealed very similar frequencies for $T 235$ in the 63 hypertensive index cases $(T 235=0.88)$, the 150 hypertensive individuals ( $T 235=0.86$ ), and the 93 normotensive controls $(T 235=0.84)$. There was no significant association with hypertension when allele frequencies from either the hypertensive index cases $\left(\chi^{2}=0.157,1\right.$ d.f, $\left.P=0.69\right)$ or the 150 unrelated hypertensives ( $\chi^{2}=0.664,1$ d.f, $P=0.41$ ) were compared with the allele frequencies in the 93 normotensive controls.

\section{Discussion}

In this study the angiotensinogen locus has been linked to human essential hypertension among African Caribbean families from St. Vincent in the West Indies. There have been difficulties confirming linkage of putative markers in other multifactorial disorders $(18,19)$. Accordingly, these data, in tandem with linkage studies in White European hypertensive populations, represent important support for an etiological role of the angiotensinogen locus in essential hypertension $(2,3)$.

Reinforcement for the linkage data arises from examination of the distribution of the $G T$ repeat alleles, using the index cases from our families or an independent cohort of unrelated hypertensives, in comparison with normotensive controls; both of which indicate a strongly positive association of this marker with hypertension. In accordance with studies in White Europeans, there is disequilibrium between several alleles of the $G T$ repeat in the African Caribbeans which might suggest that there are several etiological mutations at this locus (2). An alternative explanation could be that several of the overrepresented alleles of the $G T$ repeat are in linkage disequilibrium with a single etiological mutation.

This disequilibrium does not arise from a population stratification artifact because data from another dinucleotide repeat close to the renin gene (20) show no disequilibrium between hypertensives and normotensives in the same Vincentian population (our unpublished data). Furthermore, the younger mean age and leaner body mass index of the normotensives might be expected to dilute any disequilibrium of the $G T$ repeat between cases and controls, particularly since it is possible that some of the normotensives will track on to develop hypertension as blood pressure rises with age, in spite of our strict selection criteria. Differences in allelic representation of this marker between White Europeans $(2,3)$ and African Caribbeans are apparent from this study. It is interesting that different alleles appeared to be shared in excess and yet linkage of the angiotensinogen locus is retained in both groups $(2,3)$.

The observation that support for linkage to hypertension arises predominantly from the 46 female-female pairs may merely reflect the small number of male-male pairs in this study. The number of male pairs available for study means that results from APM method for this data set should be treated with

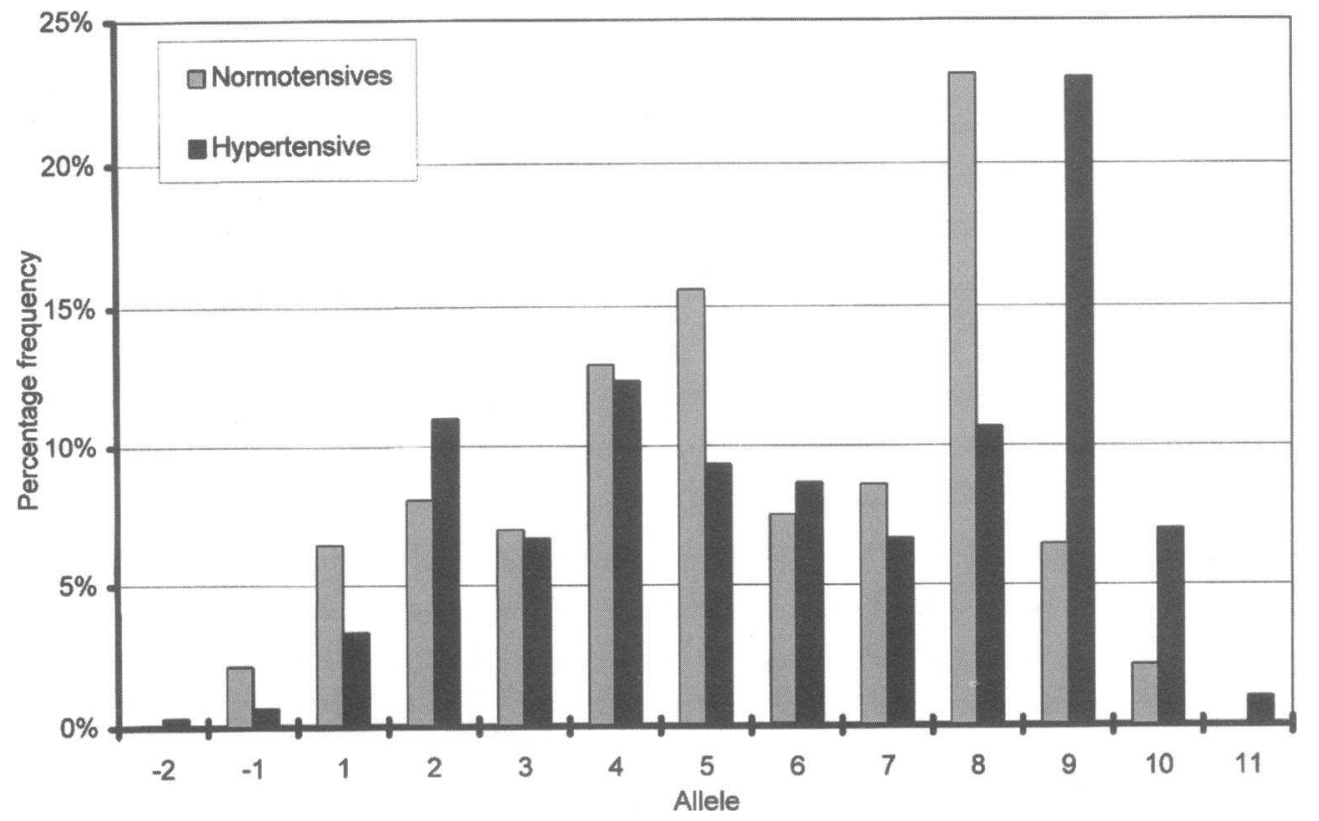

Figure 3. Comparison of the percentage of allele frequency of the $G T$ repeat in 150 unrelated hypertensives and 93 unrelated normotensives demonstrating disequilibrium in the distribution of several alleles between these groups. In particular alleles 9 and 10 are overrepresented and alleles 5 and 8 are underrepresented in the hypertensives. 
caution until the same marker has been evaluated in a larger number of male-male pairs.

The weighted $T$ statistics computed by the APM method are sensitive to control allele frequencies $(14,15)$. Some of the support for linkage in this study emerges from families sharing rare alleles in excess. The distribution of the family by family $T$ statistics is clearly positively skewed, which contrasts with the results from our previous study on White Europeans (2). Hence, relatively few families that show marked excess allele sharing contribute to the overall significant displacement of the distribution to the right. Accordingly, it will be important to confirm our observations in other populations of West African origin.

Furthermore, even though there is linkage and association of a $G T$ repeat at the angiotensinogen gene locus, this does not confirm angiotensinogen as the gene harboring susceptibility variants for hypertension. However, the observation of association between alleles of the $G T$ repeat and hypertension suggests that a susceptibility gene may lie within $\sim 100 \mathrm{~kb}$ of this marker, which makes angiotensinogen the most likely candidate.

The angiotensinogen variant $M 235 T$ is remote from angiotensin cleavage sites and its precise role remains to be clarified (3). Evidence that higher plasma levels of the gene product track with the same $T 235$ allele that associates with hypertension in White Europeans provided a putative physiological relationship between genotype and phenotype (3). However, there are now several conflicting reports of association of this variant and hypertension in White Europeans and Japanese $(2,21,22)$. Intriguingly, in this study of African Caribbeans and other investigations on populations of West African ancestry T235 is the most prevalent allele and yet no association with this disorder is demonstrated $(13,23)$.

There are several possible explanations for the disparate findings with this variant in African Caribbeans $(13,23)$. The higher prevalence of $T 235$ within populations of African origin render this polymorphism less informative than in White Europeans $(13,23)$. Furthermore, the possibility that several etiological mutations contribute to the hypertensive phenotype may account for the lack of association of M235T in some populations. Unfortunately, we were unable to store samples for determination of plasma angiotensinogen and cannot describe the relationship of plasma levels to $M 235 T$ in this study.

The African Caribbean population of St. Vincent may have genetic influences from several backgrounds including Carib Indians, Arawak Indians, and West Africans (24). Since there may be up to $25 \%$ White European gene admixture among African Americans, this raises the question of the applicability of these data to other populations of West African ancestry (25). In this study we observed that different $G T$ repeat alleles were shared in excess among the Vincentian families from those alleles we had reported in White European families at this locus. In combination with the overrepresentation of these different alleles in unrelated Vincentian hypertensives, it is highly unlikely that these observations are due to the penetrance of Caucasian genes (2).

A previous study has examined the genetic distribution of $\mathrm{ABO}$, Rhesus, and MNS blood group antigens and blood pressure in African Caribbean Vincentians, West Africans, African Americans, Carib, and Arawak populations (26). The Vincentian distribution of frequencies and blood pressure most closely relate to African Americans and West Africans. Al- though no increase in blood pressure with increasing African admixture could be demonstrated, this may well have related to the small numbers of hypertensives in that study (26).

When blood pressure levels among populations of Nigerians, African Caribbeans, and African Americans are contrasted, the blood pressure distribution demonstrates a progressive shift to the right with increasing exposure to a Western environment (4). Indeed, epidemiological surveys show greater prevalence of hypertension and in some cases a high rate of end-organ damage among African Americans (4-6). Possible explanations for this may arise from migrancy studies in Africa which show that urbanization of rural African populations is associated with a blood pressure rise which correlates positively with increased sodium excretion (26). Some workers have suggested that this might relate to genetically determined sodium sensitivity and impaired excretion of sodium by the kidney among populations of West African origin (27). Although controversial, this theory is particularly attractive in the context of the present study since cleavage of angiotensinogen by renin is the rate-limiting step in the renin-angiotensin system which exerts a major influence on sodium homeostasis (4). Nevertheless, until specific mutations which link genotype and physiology are found, this potential link must still be treated with caution.

The additional challenge is to connect our genetic data with the observations of low plasma renin activity and poor responsiveness to angiotensin converting enzyme inhibition among African populations with hypertension $(7,8)$. It is possible that recognition of the importance of the tissue-based reninangiotensin system means that plasma measurements are a crude assessment of the activity of this system (28). A further explanation might be that the angiotensinogen locus contributes less to the genetic basis of hypertension in African Caribbeans than in White Europeans. Evidence for this arises from the observation of fewer families contributing to support for linkage in this study when compared with a similar study of White Europeans (2). In both White Europeans and Vincentians these observations confirm that essential hypertension should not be regarded as a unitarian disorder and different genes may contribute to the development of hypertension in the same ethnic group. However, it is not possible to quantify the exact contribution of this locus from our studies.

The angiotensinogen locus on chromosome 1q42-43 has now been linked to human essential hypertension in populations of White Europeans and now African Caribbeans from St. Vincent and the Grenadines $(2,3)$. The exact mutations influencing disease susceptibility remain to be discovered. This study presents further important evidence for a role of this locus in the etiology of hypertension and suggests similarities in the genetic basis of this disorder in different ethnic groups.

\section{Acknowledgments}

We thank the doctors, nurses, and patients from St. Vincent and the Grenadines (especially Dr. Rampersaud and Dr. Gopinath from the Ministry of Health). The field team included Ms. S. Goodman, Dr. T. Jones, Dr. R. Sharp, Dr. C. Burton, Dr. G. Crittenden, and Dr. P. De Freitas.

This work was supported by The Joint Research Board of St. Bartholomew's Hospital, The Mason Medical Foundation, The Fellowship of Postgraduate Medicine, and The Wellcome Trust.

\section{References}

1. Williams, R. R., S. C. Hunt, S. J. Hasstedt, P. N. Hopkins, L. L. Wu, T. D. Berry, B. M. Stults, G. K. Barlow, C. Schumacher, R. P. Lifton, and J.-M. 
Lalouel. 1991. Are there interactions between genetic and environmental factors predisposing to high blood pressure? Hypertension (Dallas). 19(Suppl. 1):129-1-37.

2. Caulfield, M., P. Lavender, M. Farrall, P. Munroe, M. Lawson, P. Turner, and A. J. L. Clark. 1994. Linkage of the angiotensinogen gene to human essential hypertension. N. Engl. J. Med. 330:1629-1633.

3. Jeunemaitre, X., F. Soubrier, Y. V. Kotelevtsev, R. P. Lifton, C. S. Williams, A. Charru, S. C. Hunt, P. N. Hopkins, R. R. Williams, J. M. Lalouel, and P. Corvol. 1992. Molecular basis of human hypertension: role of angiotensinogen. Cell. 71:169-180.

4. Cooper, R. C., and C. Rotimi. 1994. Hypertension in populations of West African origin: is there a genetic predisposition. J. Hypertens. 12:215-227.

5. Whittle, J. C., P. K. Whelton, A. J. Seidler, and M. J. Klag. 1991. Does racial variation in risk factors explain black-white differences in the incidence of hypertensive end-stage renal disease? Arch. Intern. Med. 151:1359-1364.

6. Broderick, J. P., T. Brott, T. Tomsick, G. Husler, and R. Miller. 1992. The risk of subarachnoid and intracerebral haemorrhages in Blacks as compared with whites. N. Engl. J. Med. 326:733-736.

7. Anonymous. 1992. Hypertension in black and white. Lancet. 339:28-29.

8. Kaplan, N. M. 1994. Ethnic aspects of hypertension. Lancet. 344:450-452.

9. Petrie, J. C., E. T. O'Brien, W. A. Littler, M. de Swiet, P. L. Padfield, and M. J. Dillon. 1986. British Hypertension Society recommendations on blood pressure measurement. Br. Med. J. 293:611-615.

10. Kotselevtsev, Y. V., E. Clauser, P. Corvol, and F. Soubrier. 1991. Dinucleotide repeat in the human angiotensinogen gene. Nucleic Acids. Res. 19:6978.

11. Sambrook, J., E. F. Fritsch, and T. Maniatis. 1989. Molecular Cloning: A Laboratory Manual. 2nd ed. Vol 1-3. Cold Spring Harbor Laboratory, Cold Spring Harbor, NY.

12. Russ, A. P., W. Maerz, V. Ruzicka, U. Stein, and W. Grob. 1993. Rapid detection of the hypertension associated Met235 $\rightarrow$ Thr allele of the human angiotensinogen gene. Hum. Mol. Genet. 2:609-610.

13. Lifton, R. P., D. Warnock, R. T. Acton, L. Harman, and J.-M. Lalouel. 1993. High prevalence of the hypertension-associated variant T235 in African Americans. Clin. Res. 41:260A. (Abstr.)

14. Weeks, D. E., and K. Lange. 1988. The affected pedigree-member method of linkage analysis. Am. J. Hum. Genet. 42:315-326.
15. Weeks, D. E., and K. Lange. 1992. A multilocus extension of the affected pedigree-member method of linkage analysis. Am. J. Hum. Genet. 50:859-868.

6. Hall, J. M., M. K. Lee, B. Newman, J. E. Morrow, L. A. Anderson, and

M. C. King. 1990. Linkage of early-onset familial breast cancer to chromosome 17q21. Science (Wash. DC). 250:1684-1689.

17. Pericak-Vance, M. A., J. L. Bebout, P. C. Gaskell, L. H. Yamaoka, W. Y. Hung, M. J. Alberts, A. P. Walker, R. J. Bartlett, C. A. Haynes, K. A. Welsh, et al. 1991. Linkage studies in familial Alzheimer disease: evidence for chromosome 19 linkage. Am. J. Hum. Genet. 48:1034-1050.

18. Editorial. 1992. Split verdict on schizophrenia. Nat. Genet. 1:231-232.

19. Marsh, D. G., and D. A. Meyer. 1992. A major gene for allergy-fact or fancy? Nat. Genet. 2:252-254.

20. Engelstein, M., T. J. Hudson, J. M. Lane, M. K. Lee, B. Leverone, G. M. Landes, L. Peltonen, J. L. Weber, and N. C. Dracopoli. 1993. A PCR based linkage map of human chromosome 1. Genomics. 15:251-258.

21. Hata, A., C. Namikawa, M. Sasaki, K. Sato, T. Nakamura, and K. Tamura. 1994. Angiotensinogen as a risk factor for essential hypertension in Japan. $J$. Clin. Invest. 93:1285-1287.

22. Bennett, C. L., A. P. Schrader, and B. J. Morris. 1993. Cross-sectional analysis of MeT235 $\rightarrow$ Thr variant of angiotensinogen gene in severe, familia hypertension. Biochem. Biophys. Res. Commun. 197:833-839.

23. Rotimi, C. N., L. Morrison, R. S. Cooper, C. Oyejide, E. Effiong, M. Ladipo, B. Osotemihen, and R. Ward. 1993. The angiotensinogen gene in human hypertension: lack of an association amongst African Americans. Hypertension (Dallas). 24:591-594.

24. Hutchinson, J., and M. H. Crawford. 1981. Genetic determinants of blood pressure level among Black Caribs of St. Vincent. Hum. Biol. 53:453-466.

25. Chakraborty, R., M. I. Kamboh, M. Nwankwo, and R. E. Ferrel. 1992. Caucasian genes in American Blacks: new data. Am. J. Hum. Genet. 50:145155.

26. Poulter, N. R. 1989. Blood pressure in urban and rural East Africa: the Kenyan Luo migrant study. In Ethnic Factors in Health and Disease. J. K. Cruickshank and D. G. Beevers, editors. Wright, London. 61-68.

27. Grim, C. E., F. C. Luft, J. Z. Miller, G. R. Meneeely, H. D. Battarbee C. G. Hames, and L. K. Dahl. 1980. Racial differences in blood pressure in Evans county, Georgia: relationship to sodium and potassium intake and plasma renin activity. J. Chronic Dis. 33:87-94.

28. Samani, N. J. 1991. New developments in renin and hypertension. $B r$. Med. J. 302:981-982. 